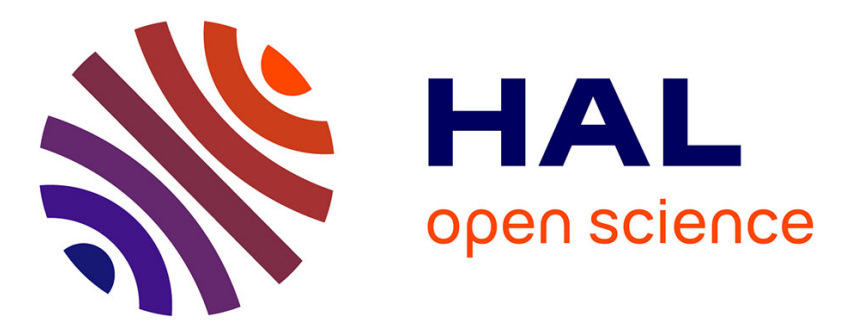

\title{
The trajectory of subboundary grooves during directional solidification of dilute alloys
}

\author{
Gabriel Faivre, Sabine Bottin-Rousseau, Silvère Akamatsu
}

\section{To cite this version:}

Gabriel Faivre, Sabine Bottin-Rousseau, Silvère Akamatsu. The trajectory of subboundary grooves during directional solidification of dilute alloys. Comptes Rendus. Physique, 2013, 14, pp.149 - 155. 10.1016/j.crhy.2013.01.003 . hal-01448374

\section{HAL Id: hal-01448374 \\ https://hal.science/hal-01448374}

Submitted on 16 Feb 2017

HAL is a multi-disciplinary open access archive for the deposit and dissemination of scientific research documents, whether they are published or not. The documents may come from teaching and research institutions in France or abroad, or from public or private research centers.
L'archive ouverte pluridisciplinaire HAL, est destinée au dépôt et à la diffusion de documents scientifiques de niveau recherche, publiés ou non, émanant des établissements d'enseignement et de recherche français ou étrangers, des laboratoires publics ou privés. 


\title{
The trajectory of subboundary grooves during directional solidification of dilute alloys.
}

\author{
G. Faivre ${ }^{\text {a }}$ S. Bottin-Rousseau ${ }^{\text {a }}$ S. Akamatsu ${ }^{a}$, \\ ${ }^{a}$ Institut des Nanosciences de Paris, UPMC, CNRS, 4 place Jussieu, 75252 Paris Cedex 05, France \\ Received $* * * * *$; accepted after revision +++++
}

\begin{abstract}
Due to their strong surface energy anisotropy, subboundaries generally do not intersect solid-melt interfaces at right angles. As a consequence, subboundary surface grooves move laterally during solidification, and thereby interact, in alloys, with the solute concentration gradients created in the liquid. We discuss the consequences of this effect during thin directional solidification at speeds $(V)$ lower than the cellular-instability threshold $V_{c}$ of the system. We show that the lateral drift of the subboundary grooves slows down (or equivalently the tilt angle of the subboundaries relative to the growth direction decreases) as $V$ increases and vanishes as $V$ approaches $V_{c}$.
\end{abstract}

\section{Résumé}

La trajectoire des sillons de sous-joints de grains en solidification directionnelle d'alliages dilués. Du fait de leur forte anisotropie capillaire, les sous-joints de grains ne coupent généralement pas les interfaces solide-liquide à angle droit. En consquence, les sillons de surface des sous-joints se déplacent latéralement en cours de solidification, et ainsi, dans les alliages, entrent en interaction avec les gradients de concentration de soluté créés dans le liquide. Nous discutons les conséquences de cet effet en solidification directionnelle d'échantillons minces à des vitesses de solidification $(V)$ inférieures au seuil d'instabilité cellulaire $V_{c}$ du système. Nous montrons que la dérive latérale des sillons des sous-joints se ralentit (ou, ce qui revient au même, l'angle d'inclinaison des sous-joints par rapport à la direction de croissance diminue) lorsque $V$ augmente et s'annule lorsque $V$ atteint $V_{c}$.

Key words: Solidification; Morphological instability ; Grain boundaries, Spatially extended systems

Mots-clés : Solidification ; Instabilité morphologique; Joints de grains, systèmes étendus spatialement

\section{Introduction}

In directional solidification, i.e., when solidification is performed at an imposed speed $V$ under a fixed unidirectional thermal gradient, the solid-melt interface of a non-faceted dilute binary alloy undergoes a morphological

Email addresses: gabriel.faivre@insp.jussieu.fr (G. Faivre), sabine.bottin-rousseau@insp.jussieu.fr (S. Bottin-Rousseau), silvere.akamatsu@insp.jussieu.fr (S. Akamatsu). 
transition at a threshold value $V_{c}$ of $V$ : the solid-melt interface is planar (it follows an isotherm) at $V<V_{c}$, and exhibits a cellular (periodically modulated) shape at $V>V_{c}$. The physical factors at play are the solute concentration gradient ahead of the interface, the applied thermal gradient $G$ and the solid-liquid surface energy $\gamma_{s l}$. The destabilizing effect exerted on the interface by the solute concentration gradient increases as $V$ increases and exceeds the stabilizing effects of the other two factors when $V$ exceeds $V_{c}[1,2]$. Coriell and Sekerka studied the influence, on this morphological transition, of isotropic grain boundaries intersecting the solid-melt interface using linearized equations for the solidification. They showed that, after a transient, grain boundary surface grooves take on a steady profile in the form of a damped sinusoid, the amplitude of which increases as $V$ increases and diverges as $V$ approaches $V_{c}$ [3].

At the melting points of alloys, high-angle grain boundaries (GBs) generally are wetted by a thin liquid film and have little surface energy anisotropy. By contrast, low-angle grain boundaries, or subboundaries (SBs) remain dry and keep, near the solid-melt interface, the high surface energy anisotropy that they have at lower temperature [4]. Therefore, when local equilibrium is reached, SBs do not intersect the solid-melt interfaces at right angle. We study here some consequences of this fact on the behavior of SBs during the solidification of dilute alloys. Let us first consider a thin (two-dimensional) directional-solidification system at rest $(V=0)$. We take the $\mathbf{x}$ axis parallel to the isotherms, the $\mathbf{z}$ axis parallel to the growth direction, the origin of $\mathbf{x}$ at the abscissa of the surface-SB junction $J$ and the origin of $\mathbf{z}$ at the isotherm that corresponds to the unperturbed planar solid-liquid interface (Fig. 1). We call $\phi$ the tilt angle of the SB with respect to $\mathbf{z}, \gamma$ the surface energy of the SB and $\gamma_{s l}$ the surface energy of the solid-liquid interface. We assume the orientation dependence of $\gamma$ to be strong and that of $\gamma_{s l}$ to be negligible. We denote the entities related to the right-hand $(x \geq 0)$ side and the left-hand $(x \leq 0)$ side of the system by the indices " $R$ " and " $L$ ", respectively. The condition of local equilibrium at $J$ is given by the Young-Herring equation

$$
\gamma_{s l}\left(\mathbf{t}_{L}+\mathbf{t}_{R}\right)+\boldsymbol{\sigma}=\mathbf{0}
$$

In this equation, $\mathbf{t}_{L}$ and $\mathbf{t}_{R}$ are the unit tangent vectors to the $R$ and $L$ flanks of the groove, respectively, and $\boldsymbol{\sigma}$ is the surface tension force or " $\sigma$ vector" of the SB defined by

$$
\boldsymbol{\sigma}=\gamma \mathbf{t}+\frac{d \gamma}{d \phi} \mathbf{n}
$$

where $\mathbf{t}$ and $\mathbf{n}$ are the unit vectors tangent and normal to the SB, respectively [5]. At rest, the solute concentration in the liquid is uniform. The condition that the surface is continuous in $J$ imposes that the shape of the groove has mirror symmetry with respect to the $\mathbf{z}$ axis. Thus $\phi$ takes a value $\phi_{a}$ (where "a" stands for "at rest") such that $\boldsymbol{\sigma}$ is parallel to $\mathbf{z}$. This angle generally is a large one owing to the strong surface energy anisotropy of SBs. Incidentally, it should be stressed that the profile of the surface groove in this "symmetrical configuration" is given by the standard formulae for a meniscus [6]: the anisotropy of the SB does not come into play, except in so far as it alters the Young angle of the surface at $J$. During solidification of a pure substance, $\phi$ would remain equal to $\phi_{a}$ independently of $V$. In this note, we show that, during directional solidification of a dilute alloy, $\phi$ deviates from $\phi_{a}$, breaking the symmetry of the groove. Most importantly, $\phi$ tends to zero as $V$ approaches $V_{c}$ irrespective of the strength of the anisotropy of the SB.

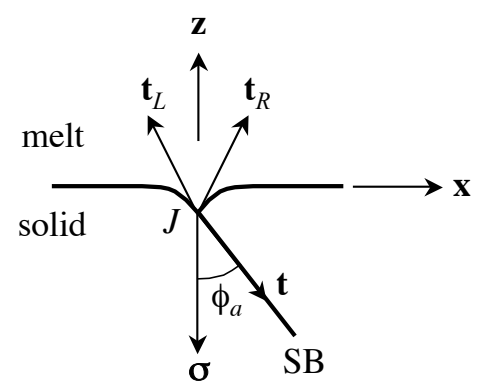

Figure 1. Symmetrical surface groove of a subboundary (SB) with a strong surface energy anisotropy at rest in a unidirectional thermal gradient. For the symbols, see the text. 


\section{The cellular instability}

The most basic model of thin directional solidification neglects the diffusion of solute in the solid, the rejection of latent heat by the growing solid and the kinetic difference in thermodynamic potentials between liquid and solid at the interface. The temperature distribution is assumed to be $\Theta=\Theta_{l i q}+G z$ ( $\Theta_{l i q}$ : liquidus temperature). The "thermal length" $l_{t h}=\Delta \Theta / D(\Delta \Theta$ : solid-liquid thermal gap; $D$ : diffusion coefficient of the solute in the liquid) is taken as a unit of length and $l_{t h}^{2} / D$ as a unit of time. $V$ and $\gamma_{s l}$ are represented by the dimensionless control parameters $\mu=l_{t h} V / D$ and $d_{o}=l_{t h}^{-1} \Delta \Theta^{-1} \Delta S_{v}^{-1} \gamma_{s l}\left(\Delta S_{v}\right.$ : entropy of fusion per unit volume), respectively. The unknowns are the profile of the solid-melt interface, denoted by $z=\zeta(x)$, and a reduced concentration field $u(x, z)$ defined such that $u(x, z=0)=1$ and $u(x, z=+\infty)=0$ in the planar steady state of the system. The equations for the solidification in the absence of grain boundary are three in number. They include the diffusion equation at $z>\zeta$

$$
\left(\partial_{x x}+\partial_{z z}+\mu \partial_{z}\right) u=\partial_{t} u
$$

and two equations at $z=\zeta$, namely, the mass conservation equation

$$
\nabla u . \mathbf{n}=-[K+(1-K) u]\left(\mu+\partial_{t} \zeta\right) \mathbf{z . n},
$$

and the Gibbs-Thomson equation

$$
\zeta=1-u+d_{o} \kappa
$$

where $\kappa$ is the curvature of the interface and $K$ is the partition coefficient. These equations admit a planar stationary solution denoted by a superscript "0", which reads

$$
\left\{\begin{array}{c}
\zeta^{0}=0 \\
u^{0}=\exp (-\mu z)
\end{array}\right.
$$

The linear stability analysis of this solution was famously performed by Mullins and Sekerka [2] and was studied in greater detail by several authors afterwards $[7,8]$. We give an outline of this analysis. A perturbed solution of the form $\zeta^{0}+\operatorname{Re}(\delta \zeta), u^{0}+\operatorname{Re}(\delta u)$, where

$$
\left\{\begin{array}{c}
\delta \zeta=Z \exp (i k x+\omega t) \\
\delta u=U \exp (-q z) \exp (i k x+\omega t),
\end{array}\right.
$$

is plugged into the equations for the solidification. The constant $Z$ is the amplitude of the surface deformation and $U$ is the amplitude of the perturbation to the concentration field. As the system under consideration is infinite in the $\mathbf{x}$ and $\mathbf{z}$ directions, the wavevector $k$ must be real, entailing that $\omega$ and $q$ are real, and $q$ must be positive. We may assume $k$ to be positive without loss of generality. Eq. (3) leads to

$$
\omega=q^{2}-\mu q+k^{2} .
$$

After linearization with respect to the perturbation, Eqs. (4) and (5) lead to a homogeneous linear system for $Z$ and $U$, which reads

$$
\left\{\begin{array}{c}
\left(\omega+K \mu^{2}\right) Z-[q-(1-K) \mu] U=0 \\
\left(\mu-1-d_{o} k^{2}\right) Z-U=0
\end{array}\right.
$$

The characteristic equation for System (9) is

$$
\omega=[q-(1-K) \mu]\left(\mu-1-d_{o} k^{2}\right)-K \mu^{2} .
$$

The concentration-to-deformation amplitude ratio is given by

$$
M=U / Z=[q-(1-K) \mu]^{-1}\left(\omega+K \mu^{2}\right) .
$$

The problem is now solved, at least implicitly. An elementary calculation leads from Eqs. (8) and (10) to an algebraic equations for $q$ as a function of $k$ (more precisely, $k^{2}$ ) and $\mu$, and then to the function $\omega(k, \mu)$. The planar solution is stable against those modes, for which $\omega<0$ and unstable against those, for which $\omega>0$. The neutral $(\omega=0)$ modes, denoted by a subscript "n", are solutions of the system of equations 


$$
\left\{\begin{array}{c}
q_{n}^{2}-\mu q_{n}+k_{n}^{2}=0, \\
{\left[q_{n}-(1-K) \mu\right]\left(\mu-1-d_{o} k_{n}^{2}\right)-K \mu^{2}=0 .}
\end{array}\right.
$$

The calculations show that neutral modes are represented by a closed curve in the $(\mu, k)$ plane. Planar solutions are stable against any perturbation outside that "neutral curve", and unstable against some set of perturbations inside it. Let $\left(\mu_{c}, k_{c}\right)$ be the lower critical point of the neutral curve. $\mu_{c}$ is the cellular-instability threshold of the system, and $k_{c}$ the wavevector of the critical mode. When $d_{o}<<1$, as is generally the case in the experiments, $k_{c} \approx(K / 2)^{1 / 3} d_{o}^{-1 / 3}$ and $\mu_{c} \approx 1+3(K / 2)^{2 / 3} d_{o}^{1 / 3}$. The speed at which $\mu=1$ is called the "constitutional supercooling" speed. It is the speed above which the solute concentration gradient overcomes the stabilizing effects of $G$, but not yet those of $\gamma_{s l}$.

\section{Isotropic grain boundary surface grooves}

We wish to calculate $\delta \zeta(x)$ and $\delta u(x, z)$ below the cellular threshold for a stationary solid-melt interface intersected by a grain boundary at $x=0$ (Fig. 2). As the emergence of the grain boundary generates a slope discontinuity of the interface, $\delta \zeta(x)$ and $\delta u(x, z)$ must be sought in the form of piecewise-defined $(R$ or $L)$ functions. Let us first consider an isotropic GB. This problem was dealt with by Coriell and Sekerka [3], as already mentioned, but we use here a more straightforward approach consisting of replacing the equations for the solidification by the linearized Eqs. (8) to (12) from the outset. Naturally, the exact solution could be found numerically using, for instance, a boundary-integral method [10]. However, our approach is likely to yield a good approximation of the exact solution in the limit of small $p$, i.e. for shallow surface grooves. Moreover, it can be used as a method for constructing trial functions for the numerical calculations.
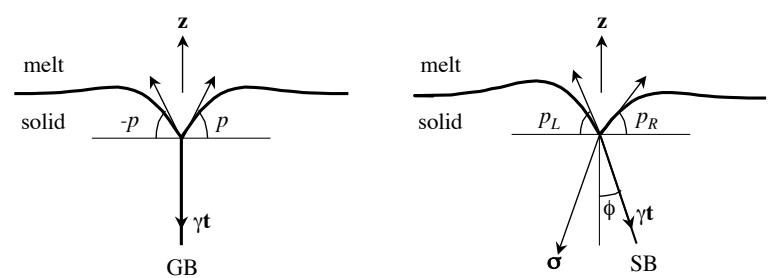

Figure 2. Steady-state surface grooves during directional solidification. Left panel: isotropic grain boundary (GB). Right panel: anisotropic subboundary (SB). The groove is drifting towards the left. The deformation of the solid-melt interface has been strongly exaggerated.

When $\mu<\mu_{c}$, the perturbation due to the GB grove must vanish at large $x$. Therefore, the wavevector in (7) must be complex, as well as the amplitudes. To be more explicit, the R-side of the interface profile should be written as

$$
\operatorname{Re}\left(\delta \zeta_{R}\right)=\exp \left(-k_{i} x\right)\left[Z_{r} \cos \left(k_{r} x\right)-Z_{i} \sin \left(k_{i} x\right)\right]
$$

with $k_{i}>0$, where the subscripts " $r$ " and " $"$ " designate real and imaginary parts, respectively. On the L-side, we have $\zeta_{L}(x)=\zeta_{R}(-x)$, or equivalently, $k_{L}=-k_{R}$. The calculation of $\delta \zeta(x)$ and $\delta u(x, z)$ at fixed $\mu$ can be performed in two stages. Firstly, $q_{n}$ and $k_{n}$ are calculated using Eqs. (12). Note that a solution with $k_{i}>0$ always exists since only $k^{2}$ appears in the equations. Secondly, the amplitudes $Z_{n}$ and $U_{n}$ are calculated using Eq. (11) and the boundary conditions at $x=0$. Figure 3 shows $k_{n}(\mu)$ calculated with values of $K$ and $d_{o}$ corresponding to the model transparent alloy $\mathrm{CBr}_{4}-C_{2} \mathrm{Cl}_{6}$ at a concentration of $0.15 \mathrm{~mol} \% \mathrm{C}_{2} \mathrm{Cl}_{6}$ under a thermal gradient of $10 \mathrm{Kmm}^{-1}[9]$. As could be expected, $k_{n}$ equals the (real) critical wavevector $k_{c}$ at $\mu=\mu_{c}$, and tends to $k_{a}=i d_{o}^{-1 / 2}$ (i.e. the purely imaginary wavevector of surface grooves at rest) as $\mu \rightarrow 0$. The evolution of $Z$ and $M$ with $\mu$ also deserves attention (right panel of Fig. 3). Note, in particular, that $\operatorname{Re}\left(M_{n}\right)$ and $\operatorname{Im}\left(M_{n}\right)$ increase very slowly as $\mu$ varies from 0 to a value of about 1 . Above this value, $\operatorname{Re}\left(M_{n}\right)$ increases almost linearly while $\operatorname{Im}\left(M_{n}\right)$ goes through a 
maximum to fall back to zero as $\mu \rightarrow \mu_{c}$. This crossover is linked to the crossing of the constitutional-supercooling threshold.

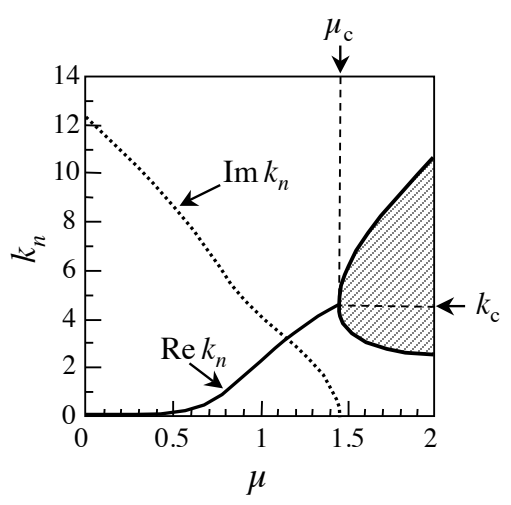

Figure 3. Subcritical $\left(\mu \leq \mu_{c}\right)$ and supercritical $\left(\mu \geq \mu_{c}\right)$ neutral modes calculated with $K=0.75$ and $d_{o}=6.5 \times 10^{-3}$. Left panel: wavevector $k_{n}$. Hatched area: instability range of the planar steady solution. Right panel: deformation amplitude $Z_{n}$ (top graph) and amplitude ratio $M_{n}$ (bottom graph).

The boundary conditions at $x=0_{+}$include the Young-Herring condition, which reads

$$
-k_{i} Z_{r}-k_{r} Z_{i}=p
$$

or, in a compact form,

$$
\operatorname{Im}\left(k_{n} Z_{n}\right)=-p
$$

where $p$ (which is independent of $\mu$ and positive) is derived from Eq. (1) with $\sigma=\gamma \mathbf{z}$. Other equations are provided by the continuity conditions. In fact, as the continuity of $\zeta$ and $u$ is already assured by symmetry, it only remains to meet the condition of continuity on $\partial u / \partial x$, which reads

$$
\operatorname{Im}\left(k_{n} M_{n} Z_{n}\right)=0 \text {. }
$$

The solution of the system formed by Eqs. (15) and (16) is

$$
Z_{n}=p \frac{M_{n}^{*}}{k_{n} M_{n i}}
$$

where $M_{n}^{*}$ is the complex conjugate of $M_{n}$. By expanding Eqs. (12) in powers of $\delta \mu=\mu-\mu_{c}$, it can be shown that $k_{i} \sim|\delta \mu|^{1 / 2}$ and $M_{i} \sim|\delta \mu|^{1 / 2}$ while $M_{n}$ tends to a real value as $\delta \mu \rightarrow 0$. Thus Eq. (17) predicts that $Z_{r}$ diverges like $|\delta \mu|^{-1 / 2}$ as $\mu \rightarrow \mu_{c}$. Simultaneously, $k_{i} Z_{r}$ and $k_{r} Z_{i}$ both tend to constant values, allowing Eq. (14) to be fulfilled until $\mu=\mu_{c}$. This means that, if the existence of a GB groove greatly affects the nature of the transient leading to the cellular instability, it leaves unchanged the value of the instability threshold, as previously noted by Coriell and Sekerka [3].

\section{Anisotropic subboundary surface grooves}

When the grain boundary that intersects the interface has a strong surface energy anisotropy, the mirror symmetry between the two sides of the surface groove is broken, and the groove travels along the surface (right panel of Fig. 2). We must therefore look for solutions to the equations for the solidification, which travel as a whole at a constant velocity $V T$, where $T=\tan \phi$, along the $\mathbf{x}$ axis. By making the change of variable $x \rightarrow x-\mu T t$ in Eq. (7), it can be seen that travelling modes (designated by a subscript " $\mathrm{t}$ " if useful) correspond to $\omega=i k \mu T$. Plotting this relation into Eqs. (8) and (10) leads to

$$
\left\{\begin{array}{c}
q_{t}^{2}-\mu q_{t}+k_{t}^{2}=i k_{t} \mu T, \\
{\left[q_{t}-(1-K) \mu\right]\left(\mu-1-d_{o} k_{t}^{2}\right)-K \mu^{2}=i k_{t} \mu T,}
\end{array}\right.
$$


which replaces the neutral-mode equations (12). The rhs terms break the mirror symmetry and entail that $k_{t L} \neq$ $-\mathrm{k}_{t R}$ and $q_{t L} \neq-\mathrm{q}_{t R}$. The calculations show that a $R-L$ pair of solutions with $k_{R i}>0$ and $k_{L i}<0$ exists at any value of $\mu$ below $\mu_{c}$ for sufficiently low values of $T$. Once $k_{D}$ and $k_{L}$ are known, the amplitudes can be determined using the Young-Herring equation and the continuity conditions at $x=0$, as explained above. However, $U_{t}$ is related to $Z_{t}$ by $U_{t}=M_{t} Z_{t}$, where $M_{t}=\left[q_{t}-(1-K) \mu\right]^{-1}\left(i \mu k_{t} T+K \mu^{2}\right)$. There are thus only four independent unknowns, for instance, the real and imaginary parts of $Z_{R}$ and $Z_{L}$. On the other hand, the Young-Herring equation now yields two independent equations (for the $R$ - and $L$-flanks of the groove, respectively), which, added to three continuity conditions, bring to five the number of conditions at $x=0$. Therefore, the problem has no solution except perhaps for a particular value of $T$, which, if it exists, depends on $\mu$. This is the central conclusion of this report. At this point, it should be noted that, as $q_{R} \neq q_{L}$, the continuity between $u_{R}$ and $u_{L}$ can only be ensured at $z=\zeta$, but not at $z>\zeta$ along the $x=0$ line. This imperfection is inherent in the linearized theory. While its quantitative consequences are unknown, its is clear that it cannot affect the fact that the lateral drift velocity is uniquely determined at given control parameters, which is a general property symmetry-broken one-dimensional out-of-equilibrium patterns $[11,12,13]$.

We shall now proceed with the calculation of $T$ as a function of $\mu$. The five above-mentioned conditions at $x=0$ are:

$$
\begin{gathered}
\operatorname{Im}\left(k_{R} Z_{R}\right)=-p_{R}, \\
\operatorname{Im}\left(k_{L} Z_{L}\right)=-p_{L}, \\
\operatorname{Re}\left(Z_{L}\right)=\operatorname{Re}\left(Z_{R}\right), \\
\operatorname{Im}\left(M_{R} Z_{R}\right)=\operatorname{Im}\left(M_{L} Z_{L}\right), \\
\operatorname{Im}\left(k_{R} M_{R} Z_{R}\right)=\operatorname{Im}\left(k_{L} M_{L} Z_{L}\right) .
\end{gathered}
$$

As mentioned, they represent a system of five linear equations for the four unknowns $Z_{R r}, Z_{R i}, Z_{L r}$ and $Z_{L i}$. Elementary but lengthy calculations show that the condition for this system to have a solution can be written in the form

$$
F_{\text {sym }}\left(p_{R}+p_{L}\right)+F_{\text {anti }}\left(p_{R}-p_{L}\right)=0
$$

where

$$
F_{s y m}=\left(\left|k_{R}\right|^{2}+\left|k_{L}\right|^{2}-2 k_{D i} k_{L i}\right) M_{D i} M_{L i}-\left(\left|M_{R}\right|^{2}+\left|M_{R}\right|^{2}-2 M_{R r} M_{L r}\right) k_{D r} k_{L r}
$$

and

$$
F_{\text {anti }}=\left(\left|k_{R}\right|^{2}-\left|k_{L}\right|^{2}\right) M_{D i} M_{L i}+\left(\left|M_{R}\right|^{2}-\left|M_{R}\right|^{2}\right) k_{D r} k_{L r}+2\left(k_{D r} M_{D r} k_{L i} M_{L i}-k_{L r} M_{L r} k_{R i} M_{R i}\right) .
$$

The functions $F_{\text {sym }}$ and $F_{\text {anti }}$ are symmetrical and antisymmetrical with respect to permutations of $R$ and $L$, respectively, hence their names. The quantities $k_{R}, k_{L}, M_{R}$ and $M_{L}$ are functions of $T$ and $\mu$ via System (18) while $p_{R}$ and $p_{L}$ are functions of $T$ via Eq. (1). Therefore Eq. (24) can be viewed as an equation for $T$ at given $\mu$. This equation can be shown to have a single solution provided that the SB has a moderate, non-singular (without facet or unstable orientation range) anisotropy. A calculated $T(\mu)$ curve is shown in Figure 4. The anisotropy function taken for the calculation was $\gamma / \gamma_{s l}=1-0.5 \times 10^{-2} \cos (4 \phi-\pi / 4)$. With this anisotropy, $p_{a} \approx 0.05$ and $T_{a} \approx \phi_{a} \approx 1.4 \times 10^{-2} \mathrm{rad}$. The values of $K$ and $d_{o}$ were the same as in Fig. 3 . It can be seen that, as $\mu$ increases from 0 to $\mu_{c}, T$ first remains close to $T_{a}$ over a large range of $\mu$, and then falls rapidly to zero. Most of the decrease occurs between $\mu=1$ and $\mu=\mu_{c}$. The right panel of Fig. 4 illustrates the amplification and shift of $\delta u$ linked to the lateral propagation of the SB groove.

Knowing that $T \rightarrow 0$ as $\mu \rightarrow \mu_{c}$, we can study in more detail the behavior of the $T(\mu)$ function in this limit. By expanding Eqs. (18), (25) and (26) in powers of $T$ it can be shown that $F_{\text {sym }} \approx 4 f_{\text {sym }}^{(0)}$ and $F_{\text {anti }} \approx 4 f_{\text {anti }}^{(1)} T$, where

$$
f_{s y m}^{(0)}=M_{n i}^{2}\left|k_{n}\right|^{2}
$$

and

$$
f_{\text {anti }}^{(1)}=k_{n r} \operatorname{Re}\left(m^{\prime} k_{n}^{*} M_{n}^{*}\right)+M_{n i} \operatorname{Im}\left(\kappa^{\prime} k_{n}^{*} M_{n}^{*}\right) .
$$

where $m^{\prime}$ and $\kappa^{\prime}$ depend on $\delta \mu=\mu-\mu_{c}$, but not on $T$. At small $T$, Eq. (1) can be written in the form $p_{R}(T) \approx$ $p_{R 0}+p_{R}^{\prime} T$ and $p_{L}(T) \approx-\left|p_{L 0}\right|+p_{L}^{\prime} T$. Plugging these expressions into Eq. (24), we obtain

$$
T \approx \frac{f_{s y m}^{(0)}\left(p_{R 0}-\left|p_{L 0}\right|\right)}{f_{\text {anti }}^{(1)}\left(p_{R 0}-\left|p_{L 0}\right|\right)+f_{\text {sym }}^{(0)}\left(p_{R}^{\prime}+p_{L}^{\prime}\right)} .
$$



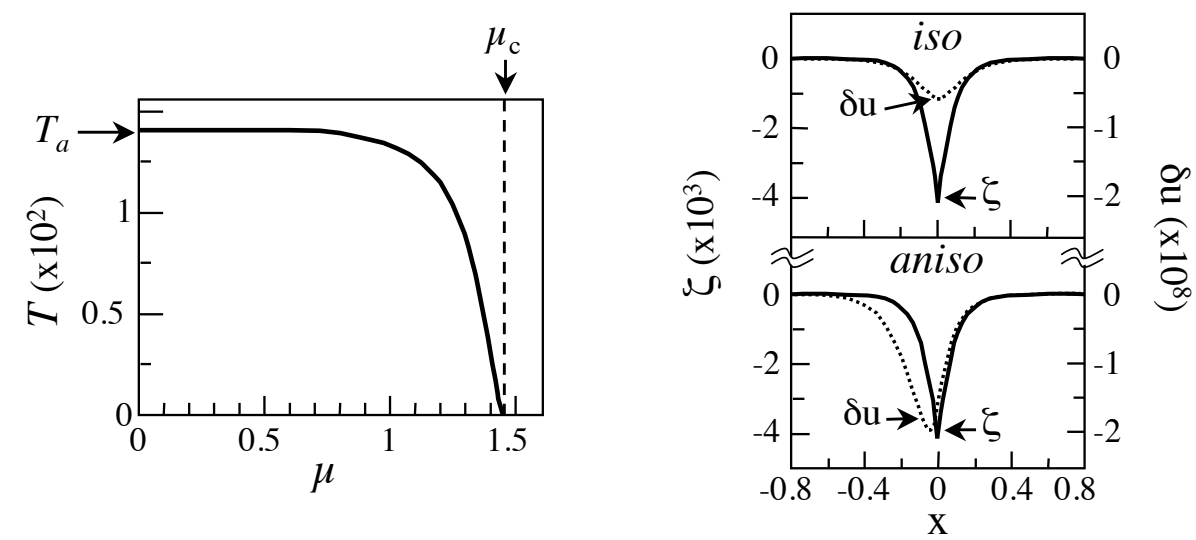

Figure 4. Left panel: Tilt angle of an anisotropic SB with respect to the direction of growth as a function of the solidification speed. For the values of the material parameters assumed in the calculation, see the text. Right panel: Profile of the surface $(\zeta)$ and perturbation to the solute distribution along the $\mathbf{x}$ axis $(\delta u)$ at $\mu=0.15$. aniso: : same SB as in the left panel. iso: isotropic $\mathrm{SB}$, everything else equal. The scales of the $x$ and $\zeta$ axes are in a ratio of $2,000: 1$

By expanding Eqs. (27) and (28) in powers of $\delta \mu$ it can be shown that $f_{\text {sym }}^{(0)} \sim|\delta \mu|$ and $f_{\text {anti }}^{(1)} \sim|\delta \mu|^{-1 / 2}$. Thus, we can finally rewrite Eq. (29) in the form

$$
T \approx-A \frac{p_{R 0}-\left|p_{L 0}\right|}{p_{R 0}+\left|p_{L 0}\right|}\left(\mu_{c}-\mu\right)^{3 / 2},
$$

where $A$ only depends on $K$ and $d_{o}$. In the limit of small $d_{o}, A$ reads

$$
A \approx 2^{4 / 3} 3^{-1 / 2} K^{-1 / 2} d_{o}^{-1 / 6}
$$

and generally is of the order of magnitude of unity. It should be noted that the ratio $\left(p_{R 0}-\left|p_{L 0}\right|\right) /\left(p_{R 0}+\left|p_{L 0}\right|\right)$ may be termed "strength of anisotropy" of the SB near $\phi=0$. Eq. 30 indicates that $\phi \rightarrow 0$ as $\mu \rightarrow \mu_{c}$ does not depend on the strength of anisotropy of the SB.

Finally, it should be reminded that the steady state of a SB-free interface in the limit of small $\mu$ is different from its state at rest. Therefore the finding (displayed in Fig.4) that, in the presence of a SB, $T \rightarrow T_{a}$ as $\mu \rightarrow 0$ is not quite obvious and requires justification. We have seen that $M_{n r} \rightarrow 0, M_{n i} \rightarrow 0, k_{n r} \rightarrow 0$ and $k_{n i} \rightarrow d_{o}^{-1 / 2}$ as $\mu \rightarrow 0$. The fact that $T$ does not tend to zero imposes that $m_{R i} \rightarrow T$ and $m_{L i} \rightarrow-T$. An elementary calculations shows that, as a consequence, $F_{\text {sym }}$ contains a term that does not tend to zero as $\mu \rightarrow 0$ (namely, the term $4 k_{n i}^{2} m_{D i} m_{L i} \rightarrow-4 d_{o}^{-1} T^{2}$ ), while $F_{a n t i}$ has none. Therefore, according to Eq. (24), the SB must approach an orientation such that $p_{R}+p_{L}=0$ as $\mu \rightarrow 0$. In other words, this proves that, at low $V$, the tilt angle of the SB is close to the symmetrical configuration corresponding to $T=T_{a}$.

\section{Conclusion}

The theory presented in this study predicts that, during the directional solidification of a dilute alloy, the tilt angle of a SB should vary from a finite value (corresponding to the symmetrical configuration of its surface grooves and therefore depending on the strength of the anisotropy of the SB) to zero as the solidification speed varies from 0 to the cellular-instability threshold of the system. More specifically, it has been established that most of the decrease in the tilt angle takes place in the interval between the constitutional-supercooling velocity and $V_{c}$, indicating that the process is mostly linked to the overcoming of the surface energy barrier by the concentration gradients ahead of the interface. The experimental data about this phenomenon are not very numerous although the question of the interaction between solute and SBs can be traced back to the origins of modern solidification science [14]. It seems to be established that the tilt angle of the SBs is essentially $V$-independent at low $V$ and that SBs run essentially parallel to the growth direction above the cellular-instability threshold [4]. However, little is known about the transition between these two extreme regimes from the experimental standpoint. We conclude with a remark about SBs in the cellular regime. Several authors have noted that, on crossing the cellular-instability threshold, the SBs become attached to inter-cellular grooves and then run parallel to $\mathbf{z}$, as mentioned. We wish to 
point out that those inter-cellular grooves, which contain a SB generally exhibit asymmetric comma-shaped profiles that contrast with the symmetrical profiles of perfect inter-cellular grooves. As explained in Fig. 5, this means that the angles between the SBs and their $\sigma$ vectors are large, which clearly supports the basic presuppositions (strong capillary anisotropy of the SBs, local equilibrium at the junctions of the SBs with the solid-melt interface) of this work.

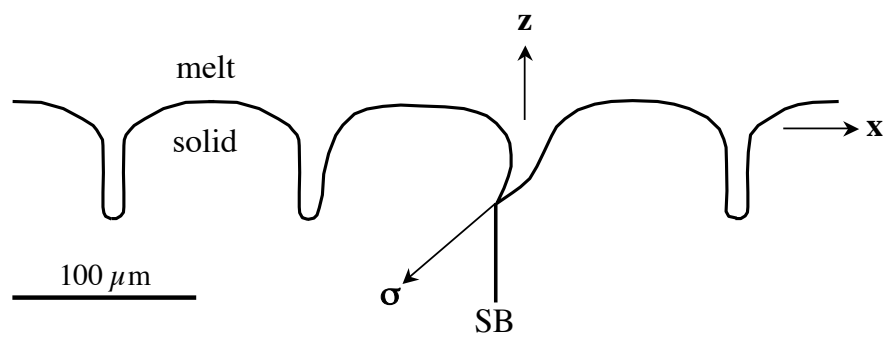

Figure 5. Directional-solidification front in a shallow-cell regime ( $V$ slightly higher than $\left.V_{c}\right)$ with a SB attached to an intercellular groove. Sketch from Fig. 4 of Ref. [4].

\section{References}

[1] W.A. Tiller and J.W. Rutter, The effect of growth conditions upon the solidification of a binary alloy, Can. J. Phys. 34 (1956) $96-121$.

[2] W.W. Mullins, R.F. Sekerka, Stability of a planar interface during solidification of a dilute binary alloy, J. Appl. Phys. 35 (1964) 444-451.

[3] S.R. Coriell, R.F. Sekerka, Morphological stability near a grain boundary groove in a solid-liquid interface during solidification of a binary alloy, J. Cryst. Growth 19 (1973) 285-293.

[4] S. Bottin-Rousseau, S. Akamatsu, G. Faivre, Formation of grain subboundaries during directional solidification below the cellular-bifurcation threshold, Phys. Rev. B 66 (2002) 054102 [1-10] .

[5] D.W. Hoffmann,J.W. Cahn, A vector thermodynamics for anisotropic surfaces, Surf. Science 31 (1972) $368-388$.

[6] L.D. Landau, E.M. Lifshitz, Statistical physics, $3^{d}$ Edition, Butterworth-Heinemann (1980).

[7] D.J. Wollkind, L.A. Segel, A nonlinear stability analysis of the freezing of a dilute binary alloy, Phil. Trans. Roy. Soc. (London) 268a (1970) 351-380.

[8] B. Caroli, C. Caroli, B. Roulet, On the emergence of one-dimensional front instabilities in directional solidification and fusion of binary mixtures, J. Phys. (France) 43 (1982) 1767-1780.

[9] J. Mergy, G. Faivre, C. Guthmann, R. Mellet, Quantitative determination of the physical parameters relevant to the thin-film directional solidification of the $\mathrm{CBr}_{4}-\mathrm{Cr}_{2} \mathrm{Clr}_{6}$ eutectic alloy, J. Cryst. Growth 134 (1993) $353-368$.

[10] J.S. Langer, L.A. Turski, Studies in the theory of interfacial stability, Acta Metall. 25 (1977) 1113-1119.

[11] P. Coullet, R.E. Goldstein and G.H. Gunaratne, Parity-breaking transitions of modulated patterns in hydrodynamics systems, Phys. Rev. Lett. 63 (1989) 1954-1957.

[12] S. Akamatsu, S. Bottin-Rousseau, M. Serefoglu, G. Faivre, A theory of thin lamellar eutectic growth with anisotropic interphase boundaries, Acta Mater 60 (2012) 3199-3205.

[13] S. Akamatsu, S. Bottin-Rousseau, M. Serefoglu, G. Faivre, Lamellar eutectic growth with anisotropic interphase boundaries: Experimental study using the rotating directional solidification methods, Acta Mater 60 (2012) 3206-3214.

[14] H.A. Atwater and B. Chalmers, The influence of impurities on the macromosaic structures of tin and lead, Can. J. Phys. 35 (1957) 208-217. 
$\mathbf{Z}$

melt

solid
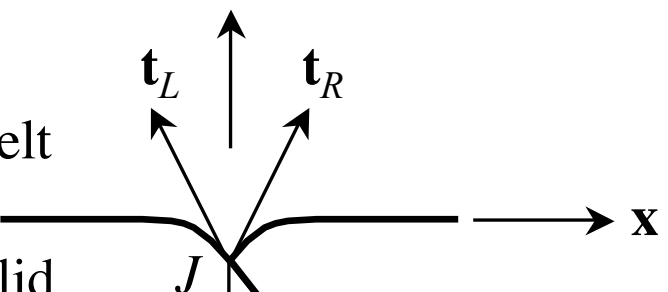


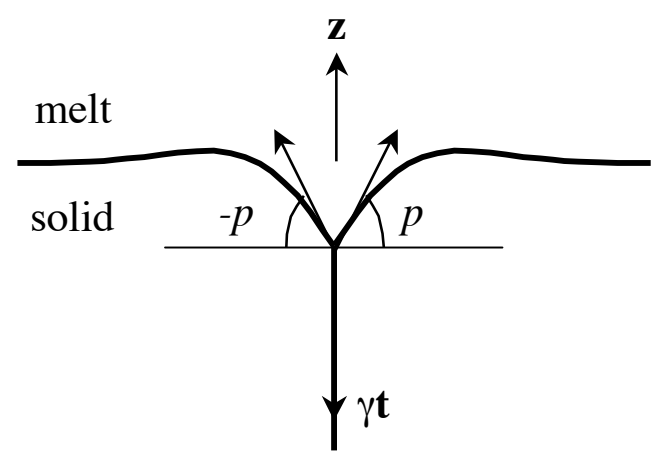

GB

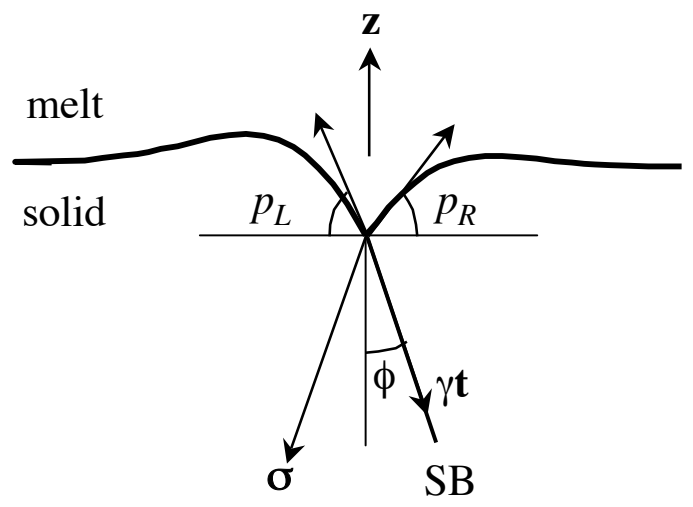




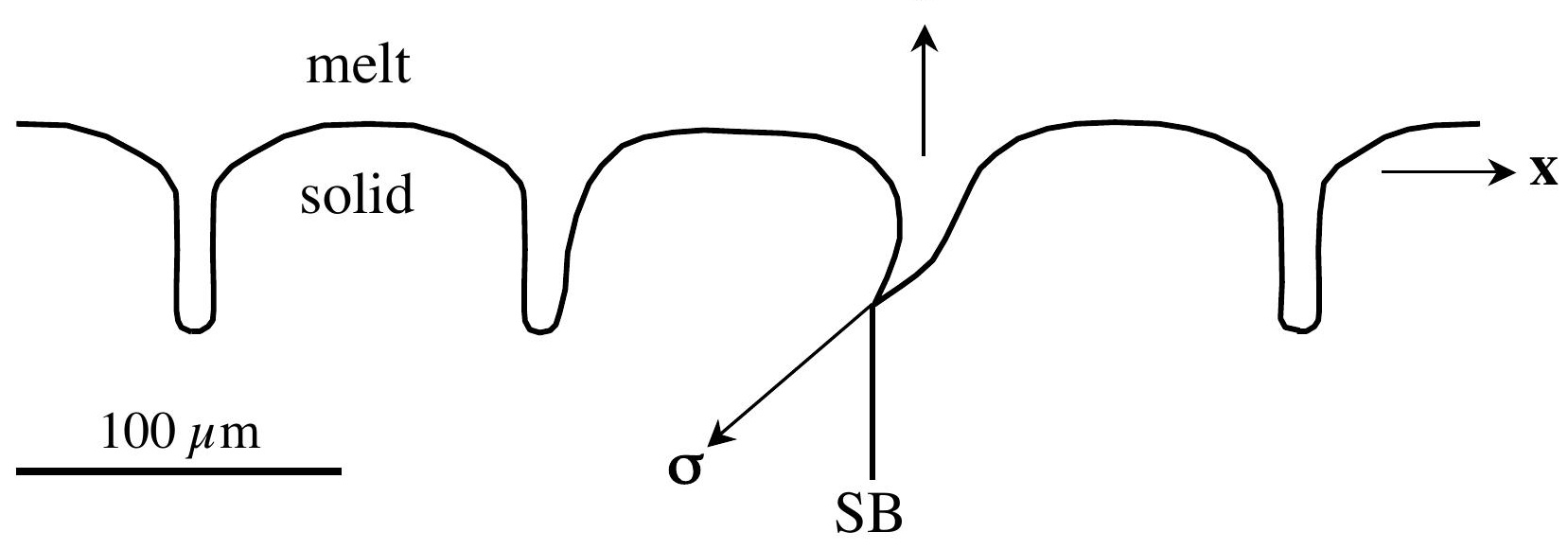

